\title{
A ORGANIZAÇÃO DA ESTRATÉGIA SAÚDE DA FAMÍLIA E ASPECTOS RELACIONADOS AO ATRASO DO DIAGNÓSTICO DA TUBERCULOSE
}

Lenilde Duarte de Sá1, Débora César de Souza Rodrigues², Anne Jaquelyne Roque Barrêto ${ }^{3}$, Annelissa Andrade Virgínio de Oliveira ${ }^{4}$, Patrícia Geórgia Oliveira Diniz Pinheiro ${ }^{5}$, Jordana de Almeida Nogueira ${ }^{6}$

\begin{abstract}
RESUMO: Objetivou-se analisar a relação entre a organização dos serviços de saúde e os fatores que influenciam no retardo do diagnóstico da tuberculose em Distritos Sanitários do Município de João Pessoa-Paraíba. Pesquisa qualitativa que envolveu seis trabalhadores da gestão de saúde - apoiadores matriciais. As informações foram coletadas mediante entrevista semiestruturada e tratadas pela técnica de Análise de Discurso. Os resultados mostram que nas equipes da Estratégia Saúde da Família não há planejamento das ações de controle da tuberculose e que prevalece a busca passiva dos sintomáticos respiratórios, demonstrando que os serviços não estão organizados para o efetivo controle da doença. Os discursos dos sujeitos também indicam que, como gestores, desconhecem a estratégia oficial de enfretamento da tuberculose e não estão sintonizados com os conceitos de promoção e vigilância da saúde. Conclui-se que o modo como está organizado o controle da tuberculose concorre para o retardo ao diagnóstico. PALAVRAS-CHAVE: Gestão em saúde; Diagnóstico tardio; Tuberculose; Programa saúde da família.

\section{THE ORGANIZATION OF THE FAMILY HEALTH STRATEGY AND ASPECTS RELATED TO DELAY IN DIAGNOSIS OF TUBERCULOSIS}

\begin{abstract}
The aim of the study was to analyze the relationship between the organization of health services and the factors which influence delay in the diagnosis of tuberculosis in the health districts of João Pessoa/Paraíba in Brazil. The research was qualitative and involved six workers in health management - support matrix. Information was collected through semi-structured interviews and processed via the technique of discourse analysis. The results show that in the Family Health Strategy teams there is no planning behind the actions for control of tuberculosis and that a passive search of people showing respiratory symptoms is prevalent, demonstrating that the services are not organized for the effective control of the illness. The subjects' discourses also indicate that, as managers, they are unaware of the official strategy for confronting tuberculosis and are not syntonized with the concepts of health promotion or health vigilance. It is concluded that the way in which the control of tuberculosis is organized contributes to delay in its diagnosis.

KEYWORDS: Health management; Late diagnosis; Tuberculosis; Family health program.

\section{LA ORGANIZACIÓN DE LA ESTRATEGIA SALUD DE LA FAMILIA Y ASPECTOS ACERCA DEL ATRASO DEL DIAGNÓSTICO DE LA TUBERCULOSIS}

RESUMEN: El objetivo fue analizar la relación entre la organización de los servicios de salud y los factores que influencían el retardo del diagnóstico de la tuberculosis en Distritos Sanitarios del Municipio de João Pessoa/Paraíba. Investigación cualitativa que involucró seis trabajadores de la administración de salud - apoyadores matriciales. Las informaciones fueron recogidas mediante entrevista semiestructurada y analizadas por medio de la técnica de Análisis del Discurso. Los resultados muestran que, en los equipos de la Estrategia Salud de la Família, no hay planeamiento de las acciones de control de la tuberculosis y que prevalece la búsqueda pasiva de los sintomáticos respiratorios, revelando que los servicios no están organizados para el efectivo control de la enfermedad. Los discursos de los sujetos también indican que, como administradores, desconocen la estrategia oficial de afrontamiento de la tuberculosis y no están sintonizados con los conceptos de promoción y vigilancia de la salud. Se concluye que el modo como se organiza el control de la tuberculosis concurre para el retardo del diagnóstico. Palabras-Clave: Administración en salud; Diagnóstico tardío; Tuberculosis; Programa salud familiar.

${ }^{1}$ Enfermeira. Doutora em Enfermagem e Saúde Pública. Professora do Departamento de Enfermagem em Saúde Pública e Psiquiatria e do Programa de Pós-Graduação em Enfermagem da Universidade Federal da Paraíba - PPGEnf UFPB.

${ }^{2}$ Enfermeira. Integrante do Grupo de Estudos e Qualificação em Tuberculose da Paraíba.

${ }^{3}$ Enfermeira. Doutoranda em Enfermagem pelo PPGEnf UFPB.

${ }^{4}$ Enfermeira. Mestranda em Enfermagem pelo PPGEnf UFPB.

${ }^{5}$ Bióloga. Mestre em Enfermagem. Coordenadora do Laboratório do Hospital Universitário da Universidade Federal de Campina Grande - PB. ${ }^{6}$ Enfermeira. Doutora em Enfermagem e Saúde Pública. Professora do Departamento de Enfermagem Clínica e do PPGEnf UFPB. 


\section{INTRODUÇÃO}

A Tuberculose (TB) encontra-se hoje em franca expansão, principalmente nos segmentos mais empobrecidos da população de países de baixa visibilidade social. Nesse milênio, é considerada a doença infectocontagiosa mais letal do mundo, o que a torna um problema de saúde pública global. A TB atinge todas as faixas etárias e seu estado de vulnerabilidade é agravado quando associado a precárias condições sociais e econômicas ${ }^{(1)}$.

No ano de 2009 surgiram cerca de 9,4 milhões de novos casos de TB em todo o mundo, estabelecendo uma taxa de 137 casos por 100.000 habitantes. É oportuno salientar que o número absoluto de casos continua aumentando de ano para ano. No referido ano, a prevalência da doença no planeta, a estimativa é de 14 milhões de $\operatorname{casos}^{(2)}$.

Concorrem para o agravamento da TB, nas últimas décadas, as desigualdades sociais e seus determinantes, a co-infecção TB/HIV (Tuberculose/Vírus da Imunodeficiência Humana), a multirresistência do bacilo de Koch, o envelhecimento da população, os movimentos migratórios, a inadequação de recursos e insuficiente apoio político no seu enfrentamento. A Organização Mundial de Saúde (OMS) decretou, em 1993, a situação de emergência global com relação à TB. Para impactar o avanço da doença, a OMS vem incentivando os países a rever seus programas de controle de TB e a adotar medidas no sentido de melhorar a condição epidemiológica da doença ${ }^{(3)}$.

No Brasil, apesar de seguir as recomendações da OMS através da positiva atuação do Programa Nacional de Controle da TB, principalmente no esforço para descentralizar as ações de controle da doença para as equipes da Estratégia Saúde da Família ${ }^{(4)}$, a TB permanece como um problema de magnitude epidemiológica e social. Estima-se que no país, em 2009, morreram 1,7 milhões de pessoas, equivalendo a uma proporção de 26 mortes por 100 mil habitantes ${ }^{(2)}$. Dentre os principais problemas que concorrem para o agravamento da TB e das mortes destaca-se o atraso do seu diagnóstico.

Parte-se do pressuposto de que a forma como estão organizados os serviços tem relação com o acesso do usuário à confirmação diagnóstica para TB. O eixo teórico orientador da análise ancora-se no conceito de organização dos serviços articulado à concepção de promoção da saúde.

Promoção da saúde é algo que vem sendo entendido nos últimos 25 anos como uma estratégia promissora para enfrentar os múltiplos problemas de saúde que afetam as populações e seus entornos. Partindo de uma concepção ampla do processo saúde-doença e de seus determinantes, a promoção da saúde propõe a articulação de saberes técnicos e populares e a mobilização de recursos insti- tucionais e comunitários, públicos e privados, para seu enfrentamento e resolução ${ }^{(5)}$.

Por sua vez, organização dos serviços de saúde referese à estrutura e ao gerenciamento da atenção à saúde. Neste estudo, privilegiar-se-á a dimensão "acesso" na avaliação da estrutura dos serviços, que será utilizada como sinônimo de acessibilidade para indicar o grau de facilidade ou dificuldade com que as pessoas obtêm cuidados de saúde. A acessibilidade é considerada como um dos principais atributos da Atenção Primária à Saúde, sendo compreendida como a ausência ou presença de barreiras financeiras, organizacionais e/ou estruturais que dificultam a obtenção do cuidado ${ }^{(6)}$.

Diante do exposto, objetivou-se com o estudo analisar a relação entre a organização dos serviços de saúde e os fatores que influenciam no retardo do diagnóstico da TB nos Distritos Sanitários I e IV, em João Pessoa-PB.

\section{METODOLOGIA}

Estudo de abordagem qualitativa, que teve como cenário os Distritos Sanitários I e IV do Município de João Pessoa$\mathrm{PB}$, selecionados por apresentarem um número significativo de casos de TB e realizarem a Terapia Diretamente Observada (TDO) pelas equipes de Saúde da Família. O Distrito Sanitário I possui em sua área de abrangência 47 equipes de Saúde da Família, enquanto o outro possui 26 equipes.

Seis trabalhadores de saúde e integrantes de equipes gestoras e dos distritos da pesquisa foram os sujeitos desse estudo, sendo quatro integrantes da equipe gestora do Distrito I e dois integrantes do IV. Todos exerciam a função de Apoiador Matricial (AM). Ser AM das Equipes de Saúde da Família foi o critério de inclusão utilizado.

Na gestão, a figura do AM é definida como a de um especialista que tem um núcleo de conhecimento e um perfil distinto daquele dos profissionais de referência, podendo agregar recursos de saber e mesmo contribuir com intervenções, aumentando a capacidade de resolver problemas de saúde da equipe primariamente responsável pelo caso ${ }^{(7)}$.

Para a coleta das informações foi utilizada a entrevista semiestruturada, realizadas entre agosto e setembro de 2009 e as respostas foram gravadas. Pela técnica de Análise de Discurso, efetuou-se um recorte do material obtido, visando destacar os fragmentos que se referiam à organização dos serviços de saúde. Na presente análise, o recorte discursivo é apresentado sob a formação discursiva central $-A$ relação da organização dos Serviços de Saúde e a obtenção do diagnóstico da TB.

Os participantes foram codificados com a sigla AM, 
seguida de números arábicos, de forma a garantir seu anonimato e atender às exigências da Resolução 196/96 do Conselho Nacional de Saúde, a qual dispõe sobre pesquisas envolvendo os seres humanos. Apoiadores matriciais AM1 e AM2 fazem parte do distrito Sanitário IV e de AM3 a AM6 são do Distrito Sanitário I. O projeto de pesquisa foi enviado ao Comitê de Ética do Centro de Ciências da Saúde da Universidade Federal da Paraíba UFPB, e aprovado sob protocolo n. 0589.

\section{RESULTADOS}

O recorte discursivo A relação da organização dos Serviços de Saúde e a obtenção do diagnóstico da TB apresenta os fragmentos dos discursos dos AM referentes à relação existente entre a organização dos serviços de saúde e a obtenção do diagnóstico da TB. Esta formação discursiva foi formada a partir de enunciados e respectivos fragmentos, a seguir apresentados (Tabela 1):

Tabela 1 - Recorte discursivo dos Apoiadores Matriciais em relação à organização dos Serviços de Saúde e a obtenção do diagnóstico da Tuberculose. João Pessoa, 2009

\section{Enunciados}

Organização do serviço na e o diagnóstico da tuberculose

Resistência dos usuários em buscar o serviço de saúde para o diagnóstico da tuberculose

\section{Fragmentos discursivos}

[...] E aí quando fazem, o escarro é encaminhado. O Distrito manda uma moto, pegar, e aí faz na Unidade todo procedimento. Passa pelo processo de informação, e aí o motoqueiro leva para o Laboratório. Quando volta o resultado, aí, começa a tomar os medicamentos [...]. (AM5) [...] o que dificulta, que eu vejo, assim, é a resistência do próprio usuário com relação a descobrir se tem a tuberculose ou não. Às vezes, ele resiste ir à Unidade de Saúde. (AM3)

[...] Para a demora tem vários fatores. Tem a questão do

Problemas estruturais relacionados aos trabalhadores transporte, a questão do laboratório que é insuficiente. da saúde e a articulação entre os pontos de atenção $[. .$.$] São dificuldades assim, são estruturais,$
são mais estruturais mesmo [...]. (AM4)

[...] a gente sabe que a maioria dos profissionais médicos são

Qualificação do profissional da saúde para a atenção à tuberculose

Busca ativa de sintomáticos respiratórios de áreas completamente diferentes do Saúde da Família. Eu acho que não se sentem responsáveis por ter que fazer isso [diagnosticar a doença]. (AM6)

[...] Na visita domiciliar, através dessa educação permanente, os Agentes de Saúde têm um olhar especifico para a questão do sintomático respiratório, de eles observarem tosse. Então,eles já têm um olhar bem atinado pra isso [...]. (AM5) E ai a enfermeira, junto com a médica, faz a visita aqueles

O protagonismo da Enfermagem na viabilização do diagnóstico da tuberculose pacientes que apresentam sintomas respiratórios [...]. A enfermeira tem sempre passado para o Agente de Saúde, e as próprias enfermeiras também com os usuários que estão sendo encaminhados às Unidades de Saúde. (AM3)

Tdentificação passiva do usuário sintomático respiratório [...] à medida que o usuário chega à Unidade apresentando sintomas (...) tanto a enfermeira quanto o médico já solicita o exame de escarro, já para se ter o diagnóstico ou não. (AM1)

[...] Tem um tipo de um programa que seria a questão do trata-

Desconhecimento dos Apoiadores Matriciais sobre a estratégia oficial de tratamento da tuberculose

mento assistido, se eunão me enganoé esse nome. Enamedida em que na área algum usuário que é portador de tuberculose, (...) ele vai tomar o remédio, sendo assistido [...]. (AM3)

Eu percebo (...) que já existe essa forma de estar promo-

Falta de planejamento para o controle da tuberculose vendo as ações para o controle da tuberculose. Não que a gente, assim, fale que tem um plano de ação [...]. (AM2) 


\section{DISCUSSÃO}

Apenas no discurso de dois dos seis AM (AM5 e AM6) que participaram do estudo há fragmentos que indicam a organização do serviço na Estratégia Saúde da Família voltada ao controle da TB, cujo enfoque recai sobre o diagnóstico da doença, ao enfatizar a presença do laboratório e um condutor para o transporte das amostras.

Nos discursos estão ausentes ações relacionadas às recomendações do Ministério da Saúde para o controle da TB, como: fazer tratamento supervisionado na unidade de saúde ou no domicílio quando indicado; fazer uma programação anual para o Programa de Controle da TB, juntamente com a Vigilância Epidemiológica do Município, estabelecendo metas a serem atingidas; fazer visita domiciliar quando necessário; treinar os recursos humanos da Unidade Básica de Saúde; realizar ações educativas junto à clientela da unidade de saúde, bem como na comunidade e divulgar os serviços prestados tanto no âmbito do serviço de saúde como na própria comunidade ${ }^{(8)}$.

Sobre os desafios que os sujeitos identificam para obtenção do diagnóstico, os fragmentos mostram problemas relacionados aos usuários e aos serviços. Com relação aos primeiros, o problema da resistência, por parte dos usuários, foi apontado nos discursos de AM3 e AM6. Percebe-se, nesses fragmentos discursivos, que a culpa pelo atraso no diagnóstico recai sobre o usuário.

O processo de utilização dos serviços de saúde é resultante da interação do comportamento do usuário que procura cuidados e do profissional que o conduz dentro do sistema de saúde. O usuário é geralmente responsável pelo primeiro contato com os serviços de saúde, enquanto os profissionais de saúde são responsáveis pelos contatos subsequentes. Portanto, são os profissionais quem têm a responsabilidade de definir o tipo e a intensidade de recursos consumidos para resolver os problemas de saúde dos usuários ${ }^{(9)}$.

Com relação aos serviços, os sujeitos AM4 e AM1 referem, em seus discursos, problemas estruturais que envolvem tanto os trabalhadores da saúde quanto a articulação entre os pontos de atenção do sistema da rede de cuidados.

A disponibilidade de serviços e de equipamentos diagnósticos e terapêuticos, a distribuição geográfica, os mecanismos de financiamento dos serviços e a sua organização em termos de estrutura, representam características do sistema que podem facilitar ou dificultar o acesso aos serviços de saúde ${ }^{(10)}$. A debilidade quantitativa e qualitativa de recursos humanos e a visão centralizada e fragmentada da organização das ações de controle da TB no sistema de saúde são barreiras que podem comprometer o acesso dos doentes às ações de diagnóstico e tratamento da TB, bem como a qualidade da interação profissional-usuário para o adequado manejo da doença $a^{(11-12)}$.

Quanto à qualificação dos profissionais de saúde, os gestores entrevistados apontam o não-preparo da equipe para lidar com a TB, principalmente do profissional médico, como se pode observar no fragmento discursivo de AM6. É importante ressaltar que, nesse fragmento, a enfermeira é citada como a profissional da equipe Saúde da Família com maior envolvimento no cuidado ao usuário com TB. O enfermeiro é fundamental na equipe da ESF, pois mantém um vínculo necessário com os usuários e, quando se aborda o cuidado com o doente de TB, este profissional amplia a eficácia das ações de saúde e favorece a participação do usuário durante a prestação do serviço ${ }^{(13)}$.

No que tange à identificação de sintomáticos respiratórios, ação imprescindível para a obtenção do diagnóstico precoce e início do tratamento, fragmentos discursivos de AM5 e AM6 mostram uma posição ambígua dos profissionais da Equipe de Saúde da Família. Uns revelam que é feita a busca ativa de casos; outros mostram que a identificação de sintomáticos se dá de forma passiva (AM1), pois passam a impressão de que os usuários devem procurar o serviço de saúde e não os serviços os identificarem no território.

A captação passiva dos casos de TB contraria a concepção da vigilância da saúde, definida como um conjunto de ações voltadas para o conhecimento, promoção, prevenção e enfrentamento sistemático de problemas de saúde, sendo estes selecionados e relativos aos fatores e condições de risco, atuais e potenciais, e aos acidentes, incapacidades e outros agravos à saúde de uma população num território definido ${ }^{(14)}$. Essa forma também contraria as orientações gerais de controle da TB, a qual informa ser o Agente Comunitário de Saúde (ACS) o responsável pela identificação de sintomáticos respiratórios na comunidade ${ }^{(8)}$.

A partir do discurso de AM3, reconhece-se a participação do ACS na identificação dos sintomáticos respiratórios, sendo também reafirmado o protagonismo do enfermeiro nessa importante ação de controle da TB. $\mathrm{O}$ ACS, integrante das equipes vinculadas à ESF, possui uma situação singular, uma vez que deve obrigatoriamente residir na área de atuação da equipe e exercer a função de elo entre a equipe e a comunidade, vivendo o cotidiano da comunidade com mais intensidade do que os outros membros da equipe de saúde ${ }^{(15)}$. 
O Plano Nacioanl de controle da TB no Brasil conta com a Estratégia Saúde da Família e o Programa de Agentes Comunitários de Saúde, com parceiros que tendem a contribuir na expansão das ações do Programa de Controle da TB, pois a família e o domicílio consistem no locus de seu trabalho ${ }^{(16)}$. Nas visitas domiciliares dos ACS está prevista a detecção de usuários sintomáticos respiratórios e contatos, principalmente nos casos bacilíferos e de crianças ${ }^{(17)}$.

Vale ressaltar que a busca ativa dos sintomáticos respiratórios é uma das prioridades do Programa Nacional de Controle da TB, atividade que deverá se constituir como uma atitude permanente e incorporada à rotina de todos os membros da equipe de saúde ${ }^{(16)}$. Reconhece-se que a busca passiva dos casos de TB potencializa o problema do atraso do diagnóstico, bem como retarda o início do tratamento.

Com relação ao tratamento da TB, o Tratamento Diretamente Observado (TDO) se caracteriza pela administração dos medicamentos por uma segunda pessoa que entrega, observa e registra a ingestão de cada dose da medicação, podendo este procedimento ser realizado na unidade de saúde, no domicílio do paciente, no hospital ou local de trabalho ${ }^{(18)}$. Ocorre que nos fragmentos discursivos os gestores não mostram conhecimento sobre a estratégia oficial para o controle da TB no Brasil, como se pode evidenciar no fragmento discursivo de AM3.

$\mathrm{Na}$ execução das ações de controle da TB, envolvendo diretamente a organização dos serviços de saúde da ESF, bem como o contexto familiar em que vive o usuário, deverão ser considerados aspectos relacionados à sua singularidade, ao desempenho da equipe de saúde e a aspectos organizacionais inerentes ao sistema de saúde, os quais venham a garantir a efetividade das ações de cuidado ao doente de TB. Desse modo, o planejamento e a avaliação dessas ações tendem a voltar-se para a promoção da saúde, tanto no plano individual quanto no coletivo.

Apesar da TB ser área estratégica de atuação da ESF, os fragmentos discursivos de AM1 e AM2 mostram não existir planejamento voltado ao controle da doença, de modo que os serviços sejam efetivos no enfretamento de um agravo que acomete grandes centros urbanos e regiões metropolitanas, como é o caso do município do estudo.

Sendo a doença em estudo considerada um problema de Saúde Pública, necessário se faz o planejamento de ações de controle voltadas à sua descoberta precoce e à celeridade em iniciar o tratamento. Nessa perspectiva, a posição dos sujeitos, aponta no sentido de fortalecer o modelo hegemônico, de caráter biomédico, uma vez que a ênfase é dada, sobretudo, ao tratamento medicamentoso. Não se observa iniciativa que, paralelamente à medicação, revele movimentos em direção ao desenvolvimento de ações intersetoriais, fato que revelaria a prática dos atores em função da mudança do modelo de atenção, sob as concepções de promoção da saúde e vigilância da saúde.

\section{CONSIDERAÇÕES FINAIS}

No que se refere às questões da relação da organização dos Serviços de Saúde com o controle da TB percebe-se que não há organização para facilitar o diagnóstico da doença.

Foi evidenciado, nesse estudo, que não se investe na busca ativa de sintomáticos respiratórios. A busca passiva aumenta as chances de contaminação dos comunicantes e retarda o diagnóstico, o que contraria a concepção de vigilância da saúde.

No que tange aos fatores que contribuem para o retardo do diagnóstico da $\mathrm{TB}$, os discursos dos gestores revelam a falta de qualificação profissional, problemas estruturais dos serviços de saúde e a demora do usuário em procurar as Unidades de Saúde da Família.

Portanto, conclui-se que a dimensão teórico-conceitual, que deveria sustentar o processo de (re)organização de ações e serviços para a garantia de acesso, controle e do cuidado ao doente de TB, mostra-se relegada a um segundo plano, seja pela falta elementar de reflexão entre a teoria e a prática ou pela opacidade do discurso resultante na incompreensão dos conceitos teóricos de vigilância da saúde e promoção da saúde, o que concorre para a permanência do modelo biomédico.

Conclui-se que a forma como o controle da TB está organizada concorre para o retardo do diagnóstico e que os AM precisam ser qualificados para o trabalho com a gestão do cuidado para os usuários com TB e às famílias.

\section{REFERÊNCIAS}

1. Santos MAPS, Albuquerque MFPM, Ximenes RAA, Silva NLCLL, Braga C, Campelo ARL, et al. Risk factors for treatment delay in pulmonary tuberculosis in Recife, Brazil. BMC Public Health. 2005;5(25):1-8.

2. World Health Organization (WHO). Global tuberculosis control: surveillance, planning, financing: WHO report. Geneva: WHO; 2010.

3. World Health Organization (WHO). Global tuberculosis control: surveillance, planning, financing: WHO report. Geneva: WHO; 2002. 
4. Giroti SKO, Belei RA, Moreno FN, Silva FS. Perfil dos pacientes com tuberculose e os fatores associados ao abandono do tratamento. Cogitare Enferm. 2010;15(2):271-7.

5. Buss PM. Promoção da saúde e qualidade de vida. Cienc Saude Colet. 2000;5(1):163-77.

6. Starfield B. Atenção primária: equilíbrio entre necessidades de saúde, serviços e tecnologia. Brasília: Ministério da Saúde/Unesco; 2002.

7. Campos GWS, Domitti AC. Apoio matricial e equipe de referência: uma metodologia para gestão do trabalho interdisciplinar em saúde. Cad Saude Publica. 2007;23(2):399-407.

8. Ministério da Saúde (Brasil). Secretaria de Vigilância em Saúde. Manual de recomendações para o controle da tuberculose no Brasil. Brasília: Ministério da Saúde; 2010.

9. Travassos C, Martins M. Uma revisão sobre os conceitos de acesso e utilização de serviços de saúde. Cad Saude Publica. 2004;20(Supl 2):S190-8.

10. Travassos C, Castro MSM. Determinantes e desigualdades sociais no acesso e na utilização de serviços de saúde. In: Escorel S, Lobato LVC, Noronha JC, Carvalho AI, organizadores. Políticas e sistema de saúde no Brasil. Rio de Janeiro: Fiocruz/CEBES; 2008. p. 215-43.

11. Wang W, Jiang Q, Abdullah ASM, Xu B. Barriers in accessing to tuberculosis care among non-residents in Shanghai: a descriptive study of delays in diagnosis. Cent Eur J Public Health. 2007;17(5):419-23.

12. Monroe AA, Gonzales RICG, Palha PF, Sassaki CM, Netto AR, Vendramini SHF, et al. Envolvimento de equipes da atenção básica à saúde no controle da tuberculose. Rev Esc Enferm USP. [Internet] 2008;42(2) [acesso em 22 nov 2010]. Disponível: http://www.scielo. br/pdf/reeusp/v42n2/a07.pdf

13. Schimith MD, Lima MADS. Acolhimento e vínculo em uma equipe do programa saúde da família. Cad Saude Publica. 2000;20(6):1487-94.

14. Teixeira CF, Paim JS, Vilasbôas AL. SUS, modelos assistenciais e vigilância da saúde. In: Rozenfeld S, organizador. Fundamentos da vigilância sanitária. Fiocruz; 2000. p. 49-60.

15. Fortes PAC, Spinetti SR. O agente comunitário de saúde e a privacidade das informações dos usuários. Cad Saude Publica. 2004;20(5):1328-33.
16. Muniz JN, Palha PF, Monroe AP, Gonzáles RC, Ruffino Netto A, Villa TCS. A incorporação da busca ativa de sintomáticos respiratórios para o controle da tuberculose na prática do agente comunitário de saúde. Cienc Saude Colet. 2005;10(2):315-21.

17. Ministério da Saúde (BR). Plano de controle da tuberculose no Brasil no período de 2001-2005. Brasília: Ministério da Saúde; 2000.

18. Muniz JN, Villa TCS, Pedersolli CE. Tratamento supervisionado no controle da tuberculose em Ribeirão Preto: novo modo de agir em saúde. Bol Pneumol Sanit. 1999;7(1):33-42.

Cogitare Enferm. 2011 Jul/Set; 16(3):437-42 